\title{
Adaptive under-actuated control for capacitive micro-machined ultrasonic transducer based on an accurate nonlinear modeling
}

\author{
Sima Darbasi • Mohammad Javad Mirzaei • Amir Musa Abazari . \\ Ghader Rezazadeh
}

Received: date / Accepted: date

\begin{abstract}
A capacitive micromachined ultrasonic transducer (CMUT) due to many benefits is being considered as an imaging and therapeutic technology recently. The critical challenge here is to stabilize the system beyond the pull-in voltage considering imposed perturbations. The CMUT system, on the other hand, has a low range of travel and it is intrinsically unstable, which can result in a pull-in phenomenon. Consequently, in order to use the CMUT systems in a variety of medical applications that require high tuning ratio, a closedloop control technique has been designed for these systems aims at increasing the maximum capacitance and enhancing tunability as well. In this study, using the closed-loop control, the resistance of micro plate against the pull-in phenomenon has been examined. Also, in the description of the system a more accurate nonlinear modeling has been considered in the presence of an under-actuated sliding model control strategy with finite convergence time. Beside, adaptive protocols with unknown upper bounds have been designed to compensate the effects of uncertainty, unmodeled dynamics and external disturbances. The performance of controller in terms of improving output pressure, stabilizing the CMUT and its robustness to imposed perturbations have been investigated. Finally, numerical simulations
\end{abstract}

\section{S. Darbasi}

Department of Electrical Engineering, Urmia University, Urmia, Iran. E-mail: st_s.darbasi@urmia.ac.ir

M.J. Mirzaei

Faculty of Electrical \& Computer Engineering, University of Tabriz, Tabriz, Iran. E-mail: mjmirzaei95@ms.tabrizu.ac.ir

A.M. Abazari, G Rezazadeh

Department of Mechanical Engineering, Urmia University, Urmia, Iran. E-mail: am.abazari@urmia.ac.ir, g.rezazadeh@urmia.ac.ir are presented to verify the usefulness and applicability of the proposed control strategy.

Keywords Nonlinear CMUT · Tunability · Distributed model · Finite-time convergence · Disturbance rejection

\section{Introduction}

In recent decades, advances in micro electro-mechanical systems (MEMS) including performance enhancement, low power consumption and low fabrication cost have attracted remarkable attention to the use of microdevices in various applications such as micromirrors [1, micro-switches [2], microresonators 3, 4, pressure sensors [5], energy harvesters [6, 7], gas sensors [8] and capacitive structures [9]. Nevertheless, among various microdevices, capacitive micromachined ultrasonic transducers due to their various benefits including reliable fabrication processes with ease and lower cost, integration with signal processing electronics, efficient performance, low impedance, higher resolution and high transduction coefficient became as an effective technology to overcome many constraints associated with traditional transducers and it can be considered as a primary candidate for next generation of transmitting and receiving ultrasound waves 1012 .

In general, the output pressure, receive sensitivity, pull-in voltage and tuning ratio are the key parameters for designing a CMUT that can be determined by the geometrical properties of the membrane and the gap size of the structure [13. A CMUT consists of a deformable plate suspended above a rigid plate with a dielectric medium between them. Applying a voltage between the two plates, causes a uniform electric field will be created within the air gap. Induced electrostatic 
force can be used to compel the deformable plate to deflect if it succeed in dealing with mechanical stiffness of the plate. and the upper plate will be compelled to deflect by electrostatic attraction forces, however, the mechanical forces exerted from the movable plate can balance this deflection. An unstable point is where electrostatic force conquer mechanical stiffness of the plate [9, 14. This point is named pull-in. After pull-in point, the upper plate will be collapsed on bottom plate which can cause damage to device. The range of stable operational displacement of capacitive structures is considered as the main distinguishing feature of such structures, typically is restricted by the pull-in instability in one-third of the capacitive gap 15. On the other hand, increasing the DC bias voltage can improve the output pressure of transmitted waves. At the pull-in voltage, the electromechanical coupling coefficient in a CMUT approaches unity which means that all the energy in one domain can be converted to the other domains [16]. It means, the maximum sensitivity is attainable if CMUT biased at a DC voltage close to its pull-in voltage [17. Further, tunability is one of the most important characteristics of micro-tunable capacitors and it is mainly depend on the pull-in instability [18. Therefore, due to the pull-in phenomenon, engineering applications have been challenged by expanding the range of the movement of the upper plate.

There are many control techniques that have been proposed to improve the performance of a CMUT and increase its tunability $19+21$. It is well-known that most of presented solutions are applied to the mechanical part of the CMUT, such as modifications in design and manufacturing processes which can make serious effects on the performance and the cost of the system, as well as creating fabrication challenges like dealing with residual stress. A contrasting view is to use closed-loop control strategies, which are preferred nowadays in various MEMS applications, are considered as one of the most effective methods for addressing the control issues. Open-loop techniques are more sensitive than closedloop approaches to external perturbations. Therefore, as open-loop approach is susceptible to external variations cannot be a suited method for MEMS applications, particularly CMUT. Moreover, when a MEMS is controlled by an open-loop system, it can be susceptible to undesirable behaviors [22, which does not allow the MEMS system to achieve high performance.

Researchers 23] through improving a boundary tension constraint technique as well as vibration control approach proved that the closed loop system can be stable strategy. Lu and Fedder in 24 proposed a voltage control approach based on the closed-loop method for effective control on top plate which increase its sta- bility range up to $60 \%$ of its initial gap. In Chan and Dutton 25] work the gap's travel scope has been raised from $30 \%$ to $90 \%$ of the initial gap. By using the proposed strategy in 26] and investigated flatness-based controller, the top plate's travel can be extended to over $90 \%$ of its initial gap without overshooting. Despite their effectiveness, closed-loop control methods have obvious disadvantages and they are difficult to apply in practice. As well, external disturbances and unmodeled dynamics play crucial roles in the performance of a CMUT system. Nonlinear closed-loop control strategies for the characterization of MEMS devices have been demonstrated to be successful in reducing and even compensating the effects of uncertainty and disturbance $27+29]$. The displacement of movable electrode of a CMUT can be controlled by a nonlinear controller so that it can deform closely to the lower fixed electrode of the CMUT which can enhance the tunability of system while preventing the pull-in instability. In spite of this, since the difference in applied voltage between moving and fixed plates highly depends on various factors, such as unpredictability in the design and imposed disturbances as well as velocity and position of the moving plate, the Controlling method which has been noticed in previous part is a challengeable strategy and not possible as a practical matter.

The sliding mode control strategy which is a robust way in control of linear and nonlinear systems, avoids the interference of external disturbances in the convergence performance of system states [30. So, nonlinear systems have extensively been investigated with the sliding mode control which provides a suitable method that represents a highly insensitive control process and it isn't influenced by uncertainties and external disturbances $31+34$. Even so traditional sliding mode control lacks satisfactory robustness during the reaching phase, integral sliding mode (ISM) control can be a promising solution 35]. According to the control law explanation considering as the main concept of the ISM control, continuous nominal control cooperates with the discontinuous control 36. With the former control law, the tracking performance of nominal plant can be guaranteed, and with the latter, the adverse influence of perturbations can be addressed 37. The advantages of the two control techniques were combined to yield a better dynamic performance. It is known that the system states will converge to the origin within a certain time when the stability of the system is guaranteed in terms of finite-time stability 38 40. Asymptotic stability, in contrast to finite-time stability, indicates that the state of a system remains constant over an infinite value of setting times. However, as a result of a finitetime control strategy, the states of the system reach 
equilibrium point in a certain time frame. Additionally, finite-time stable systems also have excellent robust response to unknown parameter changes, external perturbations imposed from outside which can be rejected well in light of mentioned approach. Based on the defined finite time integral surfaces, for the designed controller in the sliding phase it is proved that the proposed strategies in this paper can produce better results than ones in [22, 41].

This paper proposes an adaptive sliding mode controller based on the Lyapunov theory with finite convergence time, as a method in order to cope with uncertain parameters, unmodeled dynamics, and external disturbances, while preventing the pull-in instability in the CMUT system. It should be notify that the upper bounds of imposed perturbations have been considered to be unknown. This study attempts to reveal the strength and effectiveness of the suggested nonlinear controller with the aim of increasing the tunability as well as maximizing its overall maximum capacitance. Also, some beneficial capabilities of the considered nonlinear CMUT system, like output pressure with improved characteristics have been demonstrated with an accurate model.

The paper is classified as follows: Section 2 a CMUT model, required preliminaries and problem formulations. Section 3 presents affine nonlinear mathematical model. In Section 4 adaptive under-actuated control for a CMUT with finite convergence time has been designed. Section 5 includes practical example and numerical simulations to confirm and validate the theoretical results. At the end, Section 6 concludes the article.

\section{Mathematical formulations of CMUT}

This section presents an electromechanical model of a circular microplate. Due to the lack of a sufficient and an accurate Lumped Element Model (LEM) the behaviour of MEMS devices in terms of a continues model is not studied accurately [22. Hence, trying to get an accurate model in this work the mass-spring model is used accompanying the distributed model based on the plate theories. Kirchhoff thin plate theory which considering geometrical nonlinearities is an appropriate theory for analyzing the mechanical behavior of the plates [42. A schematic depicting a circular plate actuated by electrostatics is illustrated in Fig. 1. The plate with radius $R$, density $\rho$ and thickness $h$, is fully clamped in parallel above a fixed electrode with an effective distance of $g_{0}$ 43. Hamilton's principle is applied to derive the governing equation and boundary conditions of the movable plate which can be actuated by electrostatic force. The governing equation for transverse

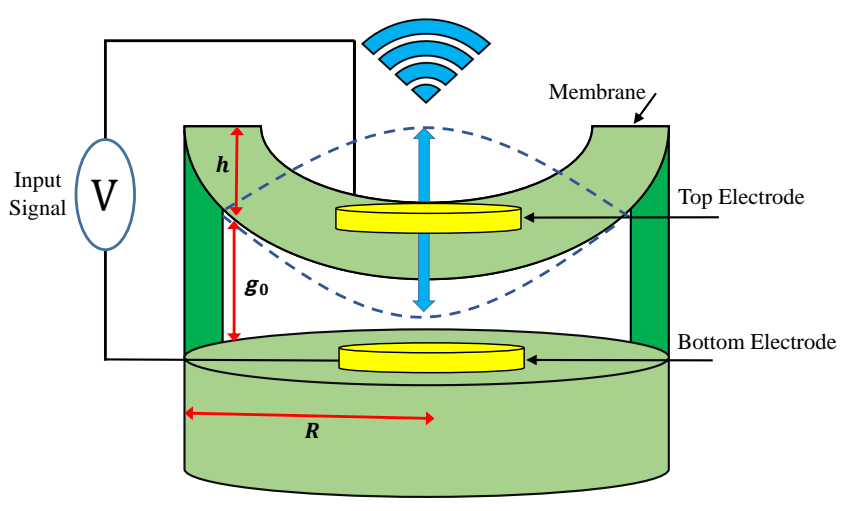

Fig. 1 schematic of a circular capacitive micromachined ultrasonic transducer (CMUT)

deformation of the movable plate, $W(r, t)$ considering an actuating voltage $V(t)$ written as

$\mathcal{D} \nabla^{4} \hat{W}+\rho h \frac{\partial^{2} \hat{W}}{\partial \hat{t}^{2}}+\mathcal{C} \frac{\partial \hat{W}}{\partial \hat{t}}=\frac{\epsilon_{0} V^{2}(t)}{2\left(g_{0}-\hat{W}\right)^{2}}$

with the following boundary conditions:

$\hat{W}(R, \hat{t})=0, \quad \frac{\partial}{\partial \hat{r}}(R, \hat{t})=0$

Also, $t$ stands for time $\epsilon_{0}$ is the permittivity of the air and $\mathcal{D}$ is the bending rigidity of the plate which is defined as follows

$\mathcal{D}=\frac{E h^{3}}{12\left(1-\nu^{2}\right)}$

where $E$ is modulus of elasticity and $\nu$ is Poisson's ratio.we assumed that there is no initial deflection on the micro plate due to residual or thermal stresses. Eq. (1) can be rewritten in a non-dimensional form which is a convenient form to work with by definition of the following non-dimensional terms:

$W=\frac{\hat{W}}{g_{0}}, r=\frac{\hat{r}}{R}, \quad t=\frac{\hat{t}}{t^{*}}, t^{*}=\sqrt{\frac{\rho h R^{4}}{\mathcal{D}}}$

By substituting the non-dimensional terms into Eq. (1), the non-dimensional form of the equation can be obtained as:

$\nabla^{4} W+\frac{\partial^{2} W}{\partial t^{2}}+\beta \frac{\partial W}{\partial t}=\frac{\lambda V^{2}(t)}{(1-W)^{2}}$

In which the appeared non-dimensional parameters are expressed as:

$\beta=\frac{\mathcal{C} R^{2}}{\mathcal{D}} \sqrt{\frac{\mathcal{D}}{\rho h}}, \quad \lambda=\frac{\epsilon_{0} R^{4}}{2 \mathcal{D}\left(g_{0}\right)^{3}}$

The Galerkin method can be used to solve the Eq. (6) . Due to non-linearity of the electrostatic force, direct 
usage of the Galerkin method is complicated. Thus, to overcome this difficulty, a non-linear term considering as forcing term in each step of the integration process, takes the value of the previous step. By selecting a sufficiently small steps, this simplification can give an accurate result. So, a solution of Eq. (1) is approximated in terms of linear combinations of finite number of suitable shape functions with time dependent coefficients $x_{i}(t)$ as follows:

$W(r, t)=\sum_{i=1}^{N} x_{i}(t) \varphi_{i}(r)$

where $\varphi_{i}(r)$ are the shape functions that satisfy the boundary conditions and $\mathrm{N}$ stands for finite number of shape functions. In this study, the shape functions are considered as:

$\varphi_{i}(r)=\cos ^{2}\left(\frac{N \pi r}{2}\right)$

After substituting Eq. (7) into Eq.(5), multiplying it by $\varphi_{j}(r)$ considering as a weight function in the Galerkin method and integrating the results from $r=0$ to 1 , a set of algebraic equations will be generated as follows:

$\sum_{i=1}^{N} \mathcal{M}_{i j} \ddot{x}_{i}(t)+\sum_{i=1}^{N} \mathcal{C}_{i j} \dot{x}_{i}(t)+\sum_{i=1}^{N} \mathcal{K}_{i j} x_{i}(t)=\mathcal{F}_{j}$

where $j=1, \ldots, N, \mathcal{M}, \mathcal{C}, \mathcal{K}$ and $\mathcal{F}$ are representing mass, damping, stiffness matrices and force vector, respectively. Because the first mode is the dominant mode 44] in vibration behaviour of the studied structure, only one mode shape, i.e. 1st mode shape is used in the present study. So, considering first mode $(N=1)$, Eq. (9) becomes:

$\mathcal{M} \ddot{x}(t)+\mathcal{C} \dot{x}(t)+\mathcal{K} x(t)=\mathcal{F}$

$\mathcal{M}=\int_{0}^{1} \varphi^{2}(r) d r, \mathcal{C}=\int_{0}^{1} \beta \varphi^{2}(r) d r$,

$\mathcal{K}=\int_{0}^{1} \nabla^{4} \varphi^{2}(r) d r, \mathcal{F}=\int_{0}^{1} \frac{\lambda V^{2}(t) \varphi(r)}{(1-x(t) \varphi(r))^{2}} d r$

A CMUT works in two energy domains: mechanical and electrical. Hence, Considering all aspects of the system when designing a controller, the equations electrical and mechanical domains need to be include in our evaluation. The mechanical equation and electrical equivalent circuit that is obtained based on Kirchhoff's Law are written as Eq. (12) and Eq. (13), respectively.

$$
\begin{aligned}
\mathcal{M} \ddot{x}(t) & +\mathcal{C} \dot{x}(t)+\mathcal{K} x(t) \\
& =\left(\frac{V^{2}(t) \epsilon_{0} R^{4}}{2 \mathcal{D}\left(g_{0}\right)^{3}}\right) \int_{0}^{1} \frac{\varphi(r)}{(1-x(t) \varphi(r))^{2}} d r
\end{aligned}
$$

$$
I_{s}(t)=\frac{1}{\Omega_{c}}\left(U_{s}(t)-U_{a c t}(t)\right)
$$

where $V(t)=\frac{Q(t) g_{0}}{\epsilon_{0} A}$ is the voltage between the upper and bottom electrodes, $I_{s}(t), U_{s}(t)$ and $U_{a c t}(t)$ are the current source, input voltage source and applied voltage respectively. Also, $A$ is the plate area, and $\Omega_{c}$ is the resistor in the external circuit 22]. The relation between charge $Q(t)$ and current $I_{s}(t)$ is equal to $I_{s}(t)=\dot{Q}(t)$. Therefore, the Eq. 12 and Eq. 13 is given as:

$$
\begin{aligned}
\mathcal{M} \ddot{x}(t) & +\mathcal{C} \dot{x}(t)+\mathcal{K} x(t) \\
& =\frac{R^{4} Q^{2}(t)}{2 \mathcal{D} g_{0} \epsilon_{0} A^{2}} \int_{0}^{1} \frac{\varphi(r)}{(1-x(t) \varphi(r))^{2}} d r \\
\dot{Q}(t)= & \frac{1}{\Omega_{c}}\left(U_{s}(t)-\frac{Q(t)\left(g_{0}-x(t)\right)}{\epsilon_{0} A}\right)
\end{aligned}
$$

To simplify the analysis, the Eq. (14) and Eq. (15) can be normalized. Normalization of charge $Q(t)$ and Voltage $U_{s}(t)$ is performed using charge $Q_{P}$ and the pull-in voltage $U_{P}$.

$q(t)=\frac{Q(t)}{Q_{P}}, \quad u_{s}(t)=\frac{U_{s}(t)}{U_{P}}$

The charge $Q_{p}$ at pull-in point, the pull-in voltage $U_{p}$ and the capacitance $C_{0}$ at initial gap are rewritten as:

$Q_{P}=\frac{3}{2} C_{0} U_{P}, \quad U_{P}=\sqrt{\frac{8 \mathcal{K} g_{0}{ }^{2}}{27 C_{0}}}, \quad C_{0}=\frac{\epsilon_{0} A}{g_{0}}$

Inserting Eq. (16) and Eq. 17) into Eq. 14 and Eq. (15) gives the normalized nominal model as follow:

$$
\begin{aligned}
& \ddot{x}(t)+2 \varrho \tilde{h}_{r} \dot{x}(t)+\tilde{h}_{r}^{2} x(t)=\int_{0}^{1} \frac{\lambda^{*} q^{2}(t) \varphi(r)}{(1-x(t) \varphi(r))^{2}} d r \\
& \dot{q}(t)+\kappa^{*}(1-x(t)) q(t)=\frac{2}{3} \kappa^{*} u(t)
\end{aligned}
$$

where $\lambda^{*}=\frac{R^{4} \mathcal{K}}{3 \mathcal{D} A}, \kappa^{*}=\frac{1}{\tilde{h}_{r} \Omega_{c} C_{0}}, \varrho=\frac{\mathcal{C}}{2 \mathcal{M} \tilde{h}_{r}}, \tilde{h}_{r}=\sqrt{\frac{\mathcal{K}}{\mathcal{M}}}$ and $u(t)$ is the input voltage. Furthermore, using Eq. (7), the 1st the dominant mode-shape and defined shape function in Eq. (8), the integral in Eq. (18) is solved as follows. Therefore, the following equations can be achieved.

$$
\begin{aligned}
\mathcal{F}(x) & =\int_{0}^{1} \frac{\cos ^{2}\left(\frac{\pi r}{2}\right)}{\left(1-x \cos ^{2}\left(\frac{\pi r}{2}\right)\right)^{2}} d r \\
& =\frac{\arctan \left(\frac{\tan \left(\frac{\pi r}{2}\right)}{\sqrt{1-x(t)}}\right)}{\pi(1-x(t))^{\frac{3}{2}}} \\
& +\left.\frac{\tan \left(\frac{\pi r}{2}\right)}{\pi(1-x(t))\left(\tan ^{2}\left(\frac{\pi r}{2}\right)\right)-x(t)+1}\right|_{0} ^{1} \\
& \Rightarrow \mathcal{F}(x)=\frac{1}{2(1-x(t))^{\frac{3}{2}}}
\end{aligned}
$$


Remark 1 According to the investigated strategies and proposed models in literature such as [22, 41, by accepting minor errors, the integral in the right hand side of Eq. 18 considering the model is neglected. However, in this work, by considering the mentioned integral the error is eliminated and consequently, a more accurate modeling is presented.

Now, we can choose the state variables of normalized model as $x(t), \dot{x}(t)$ and $q(t)$ for nonlinear state-space modeling of a CMUT. Let $\chi_{1}(t)=x(t), \chi_{2}(t)=\dot{x}(t)$, and $\chi_{3}(t)=q(t)$. The state equations can be normalized as follows:

$$
\left\{\begin{array}{l}
\dot{\chi}_{1}(t)=\chi_{2}(t) \\
\dot{\chi}_{2}=-\tilde{h}_{r}^{2} \chi_{1}(t)-2 \varrho \tilde{h}_{r} \chi_{2}(t)+\lambda^{*} \mathcal{F}\left(\chi_{1}\right) \chi_{3}^{2}(t) \\
\dot{\chi}_{3}(t)=-\kappa^{*}\left(1-\chi_{1}(t)\right) \chi_{3}(t)+\frac{2}{3} \kappa^{*} u(t) \\
Y(t)=\chi_{1}(t)
\end{array}\right.
$$

\section{Control strategy design}

In order to design a robust under-actuated control strategy and regarding the fact that applying one controller has superiority, the achieved model is transformed to an affine nonlinear model. According to the nonlinear transformation principle, the nonlinear transformation coordinate can be chosen as follows to obtain an affine nonlinear mathematical model of a CMUT.

$\mathbb{Z}=\left[\psi_{1}(t), \psi_{2}(t), \psi_{3}(t)\right]^{T} \in \Phi_{\psi}, \Phi_{\psi} \in \mathbb{R}^{3}$

where

$$
\left\{\begin{array}{l}
\psi_{1}(t)=\chi_{1}(t) \\
\psi_{2}(t)=\chi_{2}(t) \\
\psi_{3}(t)=-\tilde{h}_{r}^{2} \chi_{1}(t)-2 \varrho \tilde{h}_{r} \chi_{2}(t)+\lambda^{*} \mathcal{F}\left(\chi_{1}\right) \chi_{3}^{2}(t)
\end{array}\right.
$$

Further, it is not difficult to obtain

$$
\left\{\begin{aligned}
\dot{\psi}_{1}(t)= & \psi_{2}(t) \\
\dot{\psi}_{2}(t)= & \psi_{3}(t) \\
\dot{\psi}_{3}(t)= & -\tilde{h}_{r}^{2} \psi_{2}(t)-2 \varrho \tilde{h}_{r} \psi_{3}(t) \\
& +\lambda^{*} \dot{\mathcal{F}}\left(\psi_{1}\right) \chi_{3}^{2}(t)+2 \lambda^{*} \mathcal{F}\left(\psi_{1}\right) \chi_{3}(t) \dot{\chi}_{3}(t) \\
= & -\tilde{h}_{r}^{2} \psi_{2}(t)-2 \varrho \tilde{h}_{r} \psi_{3}(t)+\lambda^{*} \dot{\mathcal{F}}\left(\psi_{1}\right) \mathcal{G}(\mathbb{Z}) \\
& -2 \lambda^{*} \kappa^{*} \mathcal{F}\left(\psi_{1}\right)\left(1-\psi_{1}(t)\right) \mathcal{G}(\mathbb{Z}) \\
& +\frac{4}{3} \lambda^{*} \kappa^{*} \mathcal{F}\left(\psi_{1}\right) \sqrt{\mathcal{G}(\mathbb{Z})} u(t)
\end{aligned}\right.
$$

where $\mathcal{G}(\mathbb{Z})$ is defined through the following equation:

$\mathcal{G}(\mathbb{Z})=\frac{1}{\lambda^{*}} \mathcal{F}^{-1}\left(\psi_{1}\right)\left(\tilde{h}_{r}^{2} \psi_{1}(t)+2 \varrho \tilde{h}_{r} \psi_{2}(t)+\psi_{3}(t)\right)$
If $\mathcal{F}^{*}(\mathbb{Z})$ and $\mathcal{G}^{*}(\mathbb{Z})$ are considered as equations bellow,

$$
\begin{gathered}
\mathcal{F}^{*}(\mathbb{Z})=-\frac{\tilde{h}_{r}^{2}}{\theta} \psi_{2}(t)-\frac{2 \varrho \tilde{h}_{r}}{\theta} \psi_{3}(t)+\frac{\lambda^{*}}{\theta} \dot{\mathcal{F}}\left(\psi_{1}\right) \mathcal{G}(\mathbb{Z}) \\
\quad-\frac{2 \lambda^{*} \kappa^{*}}{\theta} \mathcal{F}\left(\psi_{1}\right)\left(1-\psi_{1}(t)\right) \mathcal{G}(\mathbb{Z}) \\
\mathcal{G}^{*}(\mathbb{Z})=\frac{4}{3} \lambda^{*} \kappa^{*} \mathcal{F}\left(\psi_{1}\right) \sqrt{\mathcal{G}(\mathbb{Z})}
\end{gathered}
$$

For a CMUT in the presence of unmodeled dynamics and external disturbances, the mathematical model is achieved as follows:

$$
\left\{\begin{array}{l}
\dot{\psi}_{1}(t)=\psi_{2}(t) \\
\dot{\psi}_{2}(t)=\psi_{3}(t) \\
\dot{\psi}_{3}(t)=\theta \mathcal{F}^{*}(\mathbb{Z})+\mathcal{G}^{*}(\mathbb{Z}) u(t)+\Delta \mathcal{F}(\mathbb{Z})+d_{\mathcal{C}}(t)
\end{array}\right.
$$

where $\theta$ is defined as a parameter in the system which represents the effects of existing uncertainties in the parameters for defined nonlinear transducer. Now, the CMUT with the model uncertainties, external disturbances, and unknown parameters can be stabilized with an adaptive under-actuated robust controller which is designed in the following section. The first step is to develop an estimate law for the unknown parameters. As result, to obtain convergence of the defined error $e(t)=\chi_{1}(t)-\chi_{d}$ and system trajectories to zero point, an adaptive robust control law can be applied. $\chi_{d}$ is defined as desired position of micro-plate.

Assumption 1 There are two ways in which imposed perturbations are generally modeled. The first one can be considered as unmodeled dynamics in the system, and the second one as external disturbances which are considered to be bounded and unknown. The following inequalities can be assumed to be valid if both unknown positive constants $\zeta$ and $\xi$ are introduced as follows.

$|\Delta \mathcal{F}(\mathbb{Z})| \leq \zeta, \quad\left|d_{\mathcal{C}}(t)\right|<\xi$

Following is a definition of finite time stability.

\section{Adaptive under-actuated control for CMUT with finite convergence time}

The proposed adaptive under-actuated controller is described in this section for CMUT stabilization. Also, the performance of sliding mode control strategies is generally described in this section. An appropriate sliding manifold should first be designed with the suitable dynamic properties. The second step includes developing a control signal. By controlling the system with the help of designed protocols, the states of the system will be remained on the defined sliding manifold. 


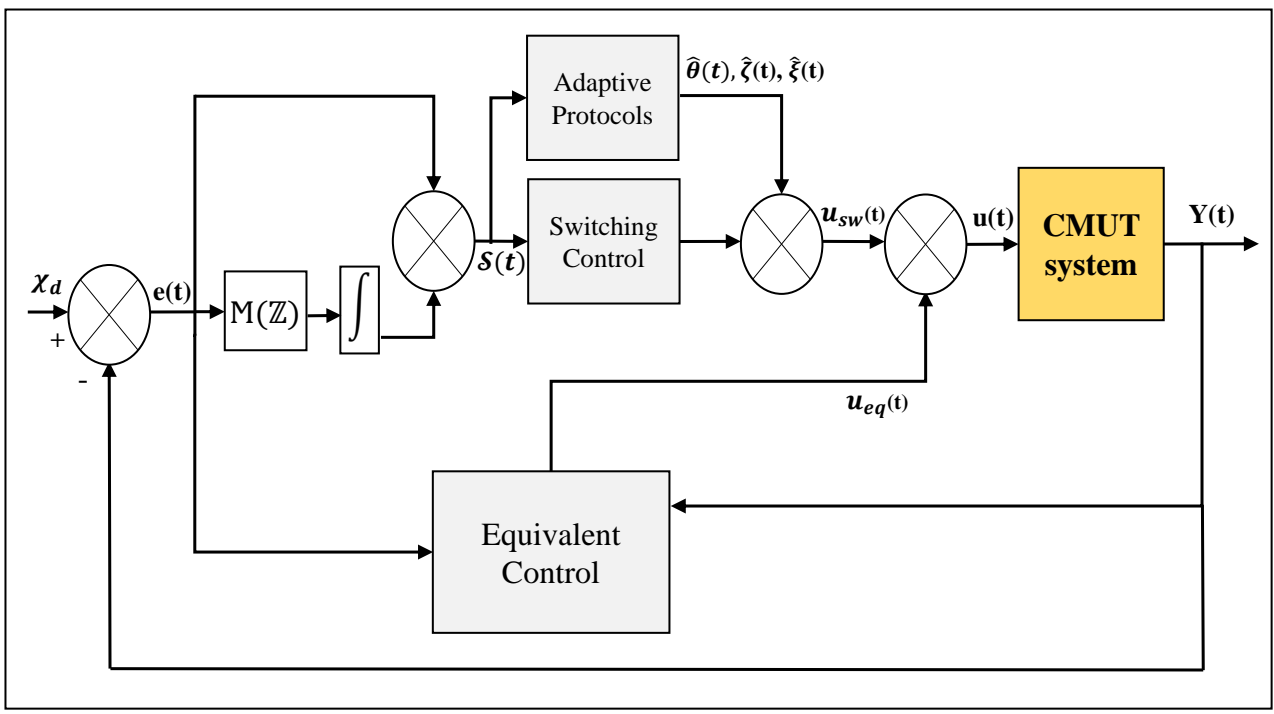

Fig. 2 Block diagram of the under-actuated adaptive integral sliding mode control strategy.

4.1 Finite-time terminal sliding surface

For the proposed of designing adaptive under-actuated controller, a nonlinear integral sliding manifold is chosen as the first design stage in this work.

$$
\begin{aligned}
& \mathcal{S}(t)=\ddot{\psi}_{1}(t)+\int_{0}^{t}\left(\rho_{3} \operatorname{sig}^{\gamma_{3}}\left(\ddot{\psi}_{1}(\tau)\right)\right. \\
&\left.+\rho_{2} \operatorname{sig}^{\gamma_{2}}\left(\dot{\psi}_{1}(\tau)\right)+\rho_{1} \operatorname{sig}^{\gamma_{1}}\left(\psi_{1}(\tau)\right)\right) d \tau \\
&=\psi_{3}(t)+\int_{0}^{t}\left(\rho_{3} \operatorname{sig}^{\gamma_{3}}\left(\psi_{3}(\tau)\right)\right. \\
&\left.\quad+\rho_{2} \operatorname{sig}^{\gamma_{2}}\left(\psi_{2}(\tau)\right)+\rho_{1} \operatorname{sig}^{\gamma_{1}}\left(\psi_{1}(\tau)\right)\right) d \tau
\end{aligned}
$$

where the constants $\rho_{i}$ and $\gamma_{i},(i=1,2,3)$ are some positives which will be defined later. $\operatorname{sig}^{\gamma}()=.|.|^{\gamma} \operatorname{sgn}($.$) ,$ and $\operatorname{sgn}($.$) represents the well-known function of sign.$ As the system states attain the sliding manifold, the following conditions should be satisfied, according to variable structure control theory.

$\mathcal{S}(t)=0, \quad \dot{\mathcal{S}}(t)=0$

According to Eq. 29] and Eq. (30), one can obtain

$$
\begin{aligned}
\dot{\mathcal{S}}(t) & =\dot{\psi}_{3}(t)+\rho_{3} \operatorname{sig}^{\gamma_{3}}\left(\psi_{3}(t)\right) \\
& +\rho_{2} \operatorname{sig}^{\gamma_{2}}\left(\psi_{2}(t)\right)+\rho_{1} \operatorname{sig}^{\gamma_{1}}\left(\psi_{1}(t)\right)=0 \\
\Rightarrow \dot{\psi}_{3}(t)=-\rho_{3} \operatorname{sig}^{\gamma_{3}}\left(\psi_{3}(t)\right) & \\
& -\rho_{2} \operatorname{sig}^{\gamma_{2}}\left(\psi_{2}(t)\right)-\rho_{1} \operatorname{sig}^{\gamma_{1}}\left(\psi_{1}(t)\right)
\end{aligned}
$$

As a result, by applying Eq. (31) to the obtained model Eq. (27), the dynamic of considered system on the de- fined sliding mode surface is achieved as follows.

$$
\left\{\begin{array}{l}
\dot{\psi}_{1}(t)=\psi_{2}(t) \\
\dot{\psi}_{2}(t)=\psi_{3}(t) \\
\dot{\psi}_{3}(t)=-\rho_{3} \operatorname{sig}^{\gamma_{3}}\left(\psi_{3}(t)\right) \\
\quad-\rho_{2} \operatorname{sig}^{\gamma_{2}}\left(\psi_{2}(t)\right)-\rho_{1} \operatorname{sig}^{\gamma_{1}}\left(\psi_{1}(t)\right)
\end{array}\right.
$$

In [45, it has been proved that for achieving the finite time stability of origin in the defined sliding mode manifold Eq. 29, the constants $\rho_{1}, \rho_{2}$, and $\rho_{3}$ should be selected such that the eigenvalues of the polynomial $\mathbb{P}_{\mathrm{w}}: m^{3}+\rho_{3} m^{2}+\rho_{2} m+\rho_{1}$ is Hurwitz, and the constants $\gamma_{1}, \gamma_{2}$, and $\gamma_{3}$ should satisfy the equality $\rho_{i-1}=\frac{\rho_{i} \rho_{i+1}}{2 \rho_{i+1}-\rho_{i}}, i=2,3$ where $\rho_{4}=1, \rho_{3}=\rho$, $\rho \in\left(1-\rho^{*}, 1\right), \rho^{*} \in(0,1)$.

Remark 2 It is observed that since the state trajectories of the achieved mathematical model of a CMUT in Eq. 27) converge to the sliding manifold Eq. 29, the overall dynamical model for the the system is transformed into Eq. (32). Due to this, the CMUT will become insensitive towards all unmodeled dynamics and disturbances, and it has been proved that the state trajectories of system Eq. (32) converge to zero in terms of finite time stability analysis.

\subsection{Adaptive sliding mode control rules}

In the presence of unmodelled dynamics, model uncertainties and external disturbances the sliding mode control signal forces the state trajectory of electrode position toward the sliding manifold defined in Eq. 29 and remains on it thereafter. Hence, an adaptive robust sliding control rule is developed to compensate 
for unknown parameters and unknown bounds. Also, Fig. 2 shows the block diagram of the adaptive underactuated sliding mode controller with updating protocols and finite time convergence for a CMUT. As discussed, the sliding mode control input should include two parts that are equivalent control $u_{e q}(t)$ and variable structure control $u_{v s}(t)$ in which control signal for the final stage is generated by:

$u(t)=1 / \mathcal{G}^{*}(\mathbb{Z})\left(u_{e q}(t)+u_{v s}(t)\right)$

This article proposes an equivalent control as follows:

$$
\begin{aligned}
u_{e q}(t) & =-\left(\hat{\theta}(t) \mathcal{F}^{*}(\mathbb{Z})+\rho_{3} \operatorname{sig}^{\gamma_{3}}\left(\psi_{3}(t)\right)\right. \\
& \left.+\rho_{2} \operatorname{sig}^{\gamma_{2}}\left(\psi_{2}(t)\right)+\rho_{1} \operatorname{sig}^{\gamma_{1}}\left(\psi_{1}(t)\right)\right)
\end{aligned}
$$

where the estimation $\hat{\theta}(t)$ is based on the unknown vector $\theta$ that according to this adaptive rule, the value is updated.

$\dot{\hat{\theta}}(t)=\mathcal{F}^{*}(\mathbb{Z}) \mathcal{S}(t), \quad \hat{\theta}\left(t_{0}\right)=\theta_{0}$

where $\theta_{0}$ is initial condition of the estimated parameter. Now, the model uncertainties and external disturbances of the system can be handled by designing of a variable structure control. So, we have developed the following vector structure signal as follow:

$u_{v s}(t)=-\left(\hat{\zeta}(t)+\hat{\xi}(t)+\pi_{v s}\right) \operatorname{sgn}(\mathcal{S}(t))$

where $\pi_{v s}>0$ is a constant gain and $\hat{\zeta}(t)$ and $\hat{\xi}(t)$ are adaptive parameters for dealing with the unknown values related to the defined upper bounds. They can be adapted using the following update laws.

$\dot{\hat{\zeta}}(t)=\zeta^{*}|\mathcal{S}(t)|, \quad \hat{\zeta}\left(t_{0}\right)=\zeta_{0}$

$\dot{\hat{\xi}}(t)=\xi^{*}|\mathcal{S}(t)|, \quad \hat{\xi}\left(t_{0}\right)=\xi_{0}$

with $\zeta^{*}>0$ and $\xi^{*}>0$ as constant values, and $\zeta_{0}, \xi_{0}$ as initial condition of estimated upper bounds. Therefore, for achieved affine nonlinear model of CMUT in Eq. (27), we propose the following control signal.

$$
\begin{aligned}
u(t)= & -1 / \mathcal{G}^{*}(\mathbb{Z})\left(\hat{\theta}(t) \mathcal{F}^{*}(\mathbb{Z})+\rho_{3} \operatorname{sig}^{\gamma_{3}}\left(\psi_{3}(t)\right)\right. \\
& +\rho_{2} \operatorname{sig}^{\gamma_{2}}\left(\psi_{2}(t)\right)+\rho_{1} \operatorname{sig}^{\gamma_{1}}\left(\psi_{1}(t)\right) \\
& \left.+\left(\hat{\zeta}(t)+\hat{\xi}(t)+\pi_{v s}\right) \operatorname{sgn}(\mathcal{S}(t))\right)
\end{aligned}
$$

Theorem 1 Consider the achieved model of a CMUT in the presence of unmodeled dynamics, external disturbances and unknown parameters of upper bounds. If Assumption 1 is satisfied and the control input is chosen as Eq. (38) As well as the adaptive laws Eq. (35) and Eq. (37), Afterwards, the system will reach $\mathcal{S}(t)=0$ in Eq. (31). Subsequently, as soon as the system states reaches to the defined manifold, the convergence of the states to the equilibrium point of origin is obtained in the sense of finite time stability.

Proof As follows is a selection of Lyapunov function candidate for the system:

$\mathcal{V}(t)=\frac{1}{2} \mathcal{S}^{2}(t)+\frac{1}{2} \tilde{\theta}^{2}(t)+\frac{1}{2 \zeta^{*}} \tilde{\zeta}^{2}(t)+\frac{1}{2 \xi^{*}} \tilde{\xi}(t)$

where $\tilde{\theta}(t)=\hat{\theta}(t)-\theta, \tilde{\zeta}(t)=\hat{\zeta}(t)-\zeta$, and $\tilde{\xi}(t)=\hat{\xi}(t)-\xi$. Then, by taking the time derivative of $\dot{\mathcal{V}}(t)$, one has

$$
\begin{aligned}
\dot{\mathcal{V}}(t)= & \mathcal{S}(t) \dot{\mathcal{S}}(t)+(\hat{\theta}(t)-\theta) \dot{\hat{\theta}} \\
& +\frac{1}{\zeta^{*}}(\hat{\zeta}(t)-\zeta) \dot{\hat{\zeta}}(t)+\frac{1}{\xi^{*}}(\hat{\xi}(t)-\xi) \dot{\hat{\xi}}(t)
\end{aligned}
$$

Introducing $\dot{\mathcal{S}}(t)$ in Eq. 31 to Eq. 40, one obtains

$$
\begin{aligned}
\dot{\mathcal{V}}(t)= & \mathcal{S}(t)\left(\dot{\psi}_{3}(t)+\rho_{3} \operatorname{sig}^{\gamma_{3}}\left(\psi_{3}(t)\right)+\rho_{2} \operatorname{sig}^{\gamma_{2}}\left(\psi_{2}(t)\right)\right. \\
& \left.+\rho_{1} \operatorname{sig}^{\gamma_{1}}\left(\psi_{1}(t)\right)\right)+(\hat{\theta}(t)-\theta) \dot{\hat{\theta}} \\
& +\frac{1}{\zeta^{*}}(\hat{\zeta}(t)-\zeta) \dot{\hat{\zeta}}(t)+\frac{1}{\xi^{*}}(\hat{\xi}(t)-\xi) \dot{\hat{\xi}}(t)
\end{aligned}
$$

Inserting system dynamics Eq. (27) into Eq. (41), one gets

$$
\begin{aligned}
\dot{\mathcal{V}}(t)= & \mathcal{S}(t)\left(\theta \mathcal{F}^{*}(\mathbb{Z})+\mathcal{G}^{*}(\mathbb{Z}) u(t)+\Delta \mathcal{F}(\mathbb{Z})\right. \\
& +d_{\mathcal{C}}(t)+\rho_{3} \operatorname{sig}^{\gamma_{3}}\left(\psi_{3}(t)\right)+\rho_{2} \operatorname{sig}^{\gamma_{2}}\left(\psi_{2}(t)\right) \\
& \left.+\rho_{1} \operatorname{sig}^{\gamma_{1}}\left(\psi_{1}(t)\right)\right)+(\hat{\theta}(t)-\theta) \dot{\hat{\theta}} \\
& +\frac{1}{\zeta^{*}}(\hat{\zeta}(t)-\zeta) \dot{\hat{\zeta}}(t)+\frac{1}{\xi^{*}}(\hat{\xi}(t)-\xi) \dot{\hat{\xi}}(t)
\end{aligned}
$$

It is clear that

$$
\begin{aligned}
\dot{\mathcal{V}}(t) \leq & \mathcal{S}(t)\left(\theta \mathcal{F}^{*}(\mathbb{Z})+\mathcal{G}^{*}(\mathbb{Z}) u(t)+\rho_{3} \operatorname{sig}^{\gamma_{3}}\left(\psi_{3}(t)\right)\right. \\
& \left.+\rho_{2} \operatorname{sig}^{\gamma_{2}}\left(\psi_{2}(t)\right)+\rho_{1} \operatorname{sig}^{\gamma_{1}}\left(\psi_{1}(t)\right)\right) \\
& +|\mathcal{S}(t)|\left(|\Delta \mathcal{F}(\mathbb{Z})|+\left|d_{\mathcal{C}}(t)\right|\right)+(\hat{\theta}(t)-\theta) \dot{\hat{\theta}} \\
& +\frac{1}{\zeta^{*}}(\hat{\zeta}(t)-\zeta) \dot{\hat{\zeta}}(t)+\frac{1}{\xi^{*}}(\hat{\xi}(t)-\xi) \dot{\hat{\xi}}(t)
\end{aligned}
$$

According to the assumption 1 of inequality Eq. 28 and the update rules Eq. (35) and Eq. (37), it is given:

$$
\begin{aligned}
\dot{\mathcal{V}}(t) \leq & \mathcal{S}(t)\left(\theta \mathcal{F}^{*}(\mathbb{Z})+\mathcal{G}^{*}(\mathbb{Z}) u(t)+\rho_{3} \operatorname{sig}^{\gamma_{3}}\left(\psi_{3}(t)\right)\right. \\
& \left.+\rho_{2} \operatorname{sig}^{\gamma_{2}}\left(\psi_{2}(t)\right)+\rho_{1} \operatorname{sig}^{\gamma_{1}}\left(\psi_{1}(t)\right)\right) \\
+ & (\hat{\theta}(t)-\theta) \mathcal{F}^{*}(\mathbb{Z}) \mathcal{S}(t)+\hat{\zeta}(t)|\mathcal{S}(t)|+\hat{\xi}(t)|\mathcal{S}(t)|
\end{aligned}
$$


Table 1 Geometrical and material properties of the movable plate.

\begin{tabular}{ccc}
\hline Symbol & Quantity & Value \\
\hline $\mathrm{R}$ & Radius & $100(\mu \mathrm{m})$ \\
$\mathrm{h}$ & Thickness & $1(\mu \mathrm{m})$ \\
$\epsilon_{0}$ & Permittivity of air & $8.8541878(\mathrm{pF} / \mathrm{m})$ \\
$\mathrm{E}$ & Young's modulus & $169(\mathrm{GPa})$ \\
$\nu$ & Poisson's ratio & 0.3 \\
$g_{0}$ & Initial gap & $1(\mu \mathrm{m})$ \\
$\rho$ & Density & $2330\left(\mathrm{~kg} / \mathrm{m}^{3}\right)$
\end{tabular}

When Eq. 44 is simplified and the control signal Eq. (38) is substituted into it, it is obtained as:

$$
\begin{aligned}
& \dot{\mathcal{V}}(t) \leq \mathcal{S}(t)\left(-\hat{\theta}(t) \mathcal{F}^{*}(\mathbb{Z})-\rho_{3} \operatorname{sig}^{\gamma_{3}}\left(\psi_{3}(t)\right)\right. \\
&- \rho_{2} \operatorname{sig}^{\gamma_{2}}\left(\psi_{2}(t)\right)-\rho_{1} \operatorname{sig}^{\gamma_{1}}\left(\psi_{1}(t)\right) \\
&-\left(\hat{\zeta}(t)+\hat{\xi}(t)+\pi_{v s}\right) \operatorname{sgn}(\mathcal{S}(t))+\rho_{3} \operatorname{sig}^{\gamma_{3}}\left(\psi_{3}(t)\right) \\
&\left.+\rho_{2} \operatorname{sig}^{\gamma_{2}}\left(\psi_{2}(t)\right)+\rho_{1} \operatorname{sig}^{\gamma_{1}}\left(\psi_{1}(t)\right)\right) \\
&+\hat{\theta}(t) \mathcal{F}^{*}(\mathbb{Z}) \mathcal{S}(t)+\hat{\zeta}(t)|\mathcal{S}(t)|+\hat{\xi}(t)|\mathcal{S}(t)| \\
& \leq-\pi_{v s}|\mathcal{S}(t)|
\end{aligned}
$$

Consequently, the Lyapunov stability ensures that the system's states Eq. (27) are converged to $\mathcal{S}(t)=0$. As a consequence, the system states converge to the equilibrium point of origin along the defined manifold in terms of finite-time stability. Proof then becomes complete.

\section{Simulation results}

In this section, the obtained results are presented and discussed. In the first part, the modeling results of this study is validated by means of comparing with [46]. In the second part, through considering uncertainties and external disturbance, the strength and capabilities of proposed controller is demonstrated for $\chi_{d}=0.95$. Then, various values for $\chi_{d}$ are analysed. In the last part, the Table 1 summarizes the geometric and physical properties of the modelled CMUT.

In order to validate the result of this study, the pullin voltage is compared with [46]. However, there is a negligible difference between the values of pull-in voltage shown in Fig. 3 the results reveal that the applied numerical method works well for the presented model.

For a tracking value of $\chi_{d}=0.95$, and considering the control parameters as follows: $\rho_{1}=6, \rho_{2}=12$, $\rho_{3}=8, \gamma_{1}=7 / 16, \gamma_{2}=7 / 13, \gamma_{3}=7 / 10, \pi_{v s}=0.5$, $\zeta^{*}=1$ and $\xi^{*}=1$, Fig. 4 and Fig. 5 are presented to demonstrate the potential of the designed controller for adjusting the dynamic response of the CMUT with the accurate nonlinear modeling. Hence, considering $\chi_{d}=0.95$, the time history of the displacement and

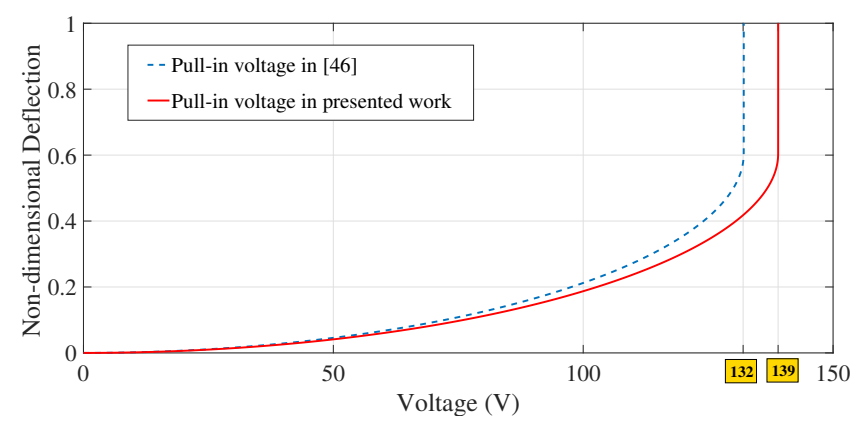

Fig. 3 Pull-in voltage of this study vs [46].

the applied voltage are shown in Fig. 4 and b respectively. In fact, it can be inferred that with a distance of $0.05 \mu \mathrm{m}$ between the movable and fixed electrodes, At steady-state, the corresponding dynamic error will be zero. According to the CMUT voltage time-history, the applied voltage decreases from zero to $-5(\mathrm{~V})$, afterwards, it increases to $10(\mathrm{~V})$. A steady-state value of $0.1(\mathrm{~V})$ is reached when the function again smoothly decreases. In addition, the coefficients of adaptation $\theta$, $\zeta, \xi$ and capacitance variation versus time are plotted in Fig 5 and b respectively. It is clear, with presence of controller the amount of the capacitance is maximized to $\simeq 6 \times 10^{-12}(F)$ and all uncertain parameters in the system are increased with time and attained their respective constant values. As Fig. 4 and 5 , showed with using a controller, the CMUT can follow a given path
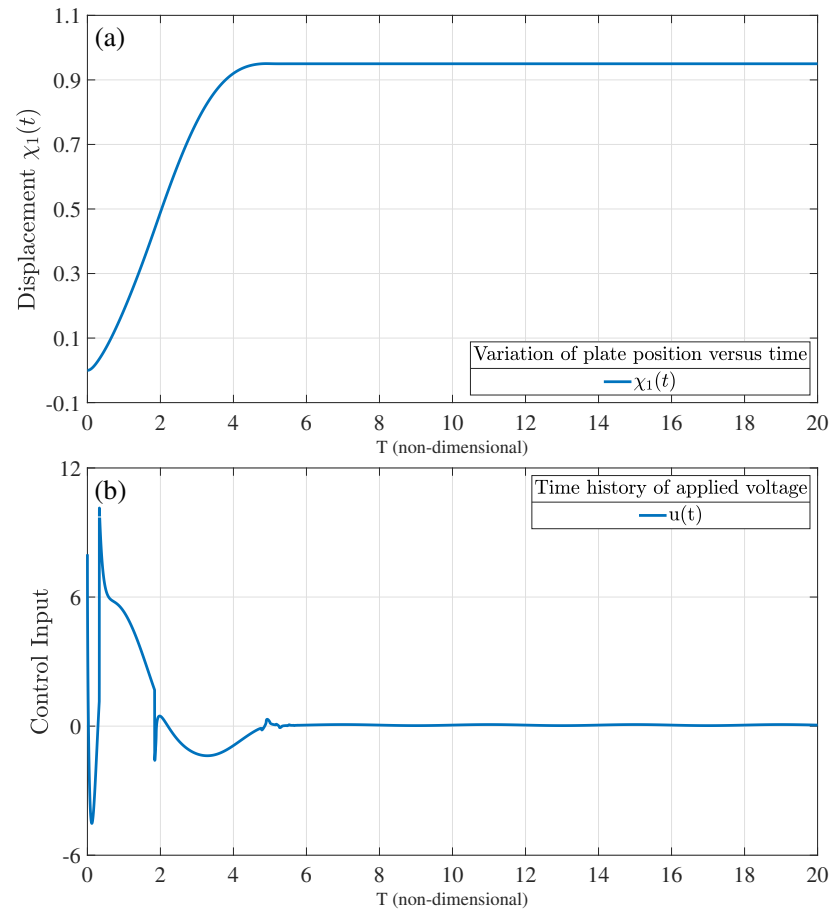

Fig. 4 The variation of a) displacement and b) control input versus time considering $\chi_{d}=0.95$. 
with a reasonable level of accuracy. The CMUT may also lead to premature dynamic pull-in if it overshoots. However, a designed adaptive under-actuated sliding mode controller doesn't exhibit this behavior in the achieved results for $\chi_{d}=0.95$, in the proposed strategy to prevent any pull-in effect and considering safe margin along with robust characteristics of the controller against parameters variations, the desired path is considered to have values not exceeding 0.9 .

In Fig. 6 and 7 considering the various values of $\chi_{d}$ the time history of displacement, error between plate position and desired value, the applied voltage and sliding surfaces are presented, respectively. In comparison with previous works [22, 41, the proposed strategy in this paper is not affected by overshooting problem. As shown in Fig. 6a and b, this problem is not observed in the behavior of the plate deflection and presented error using the designed adaptive controller with finite time convergence. The applied voltage versus time is shown in Fig. 77. The initial voltage levels for all $\chi_{d} s$ are between $-15 \mathrm{~V}$ to $15 \mathrm{~V}$ and as time elapses, they converges to around zero. Also, in $7 \mathrm{~b}$ time history of defined finite time sliding surfaces demonstrates that sliding manifolds are converged to zero.

At the end, its worth to mention that control of a CMUT is performed in order to guide the upper electrode to be in close proximity to the fixed electrode in order to enhance $C_{\max }$ value and thus the tunability
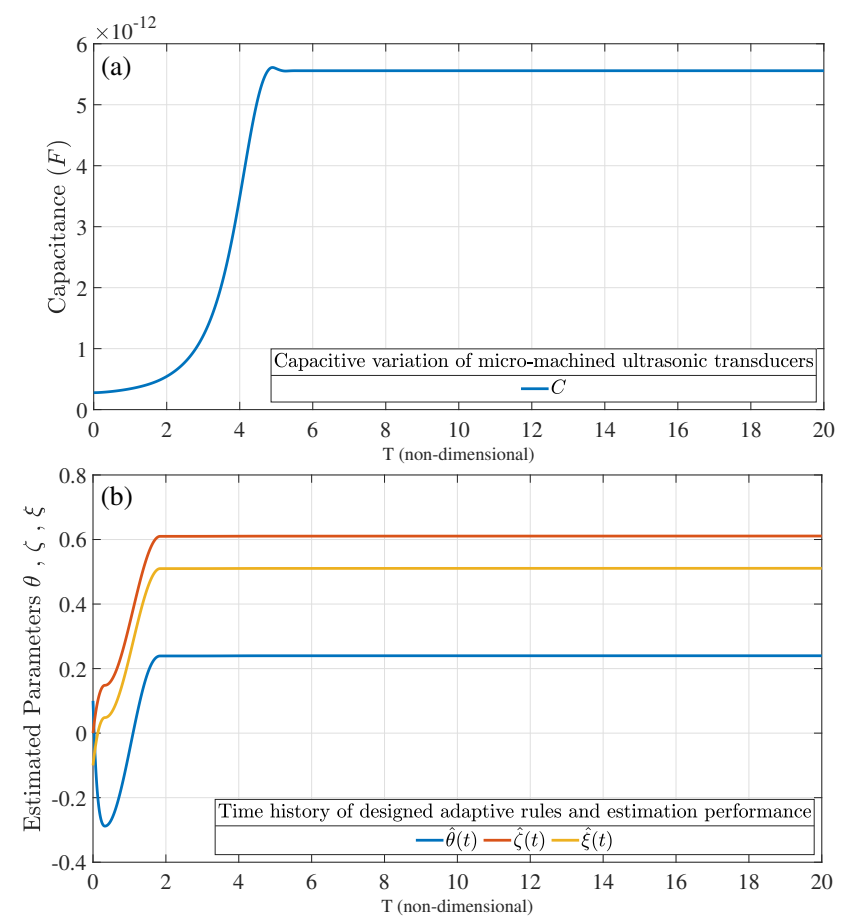

Fig. 5 The variation of a) capacitance and b) adaptive rules versus time considering $\chi_{d}=0.95$.
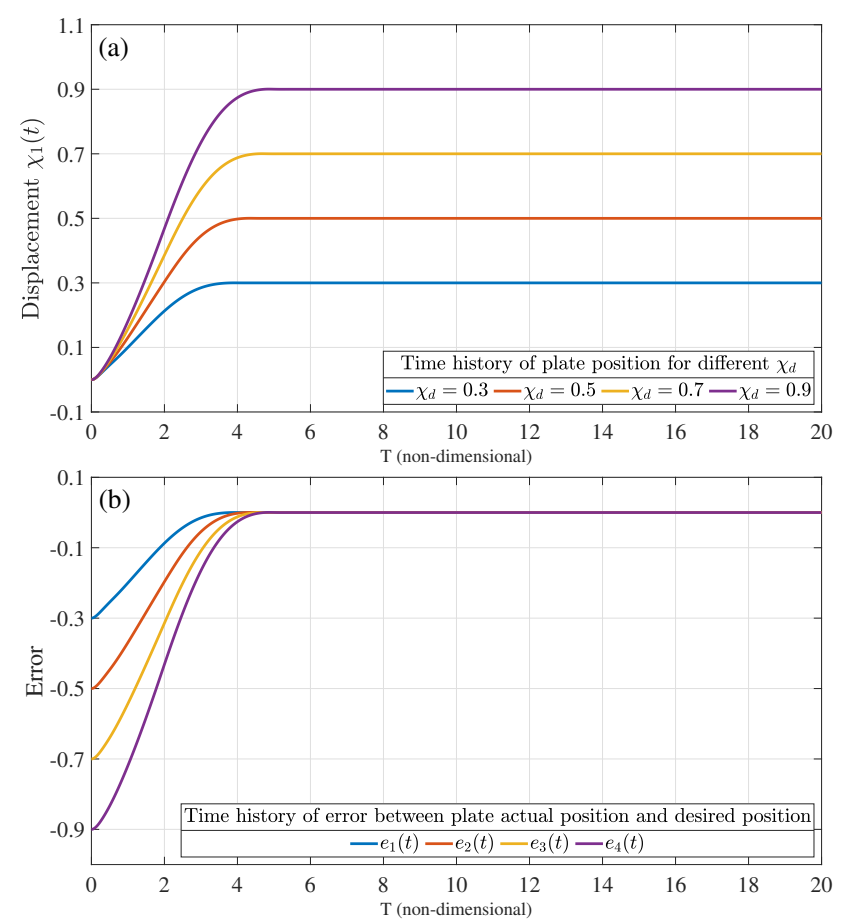

Fig. 6 The variation of a) displacement and b) error versus time for various values of $\chi_{d}$

Table 2 The values of tuning ratio, maximum capacitance and tunability through implementing the proposed controller for various values of $\chi_{d}\left(C_{\min }=2.779 \times 10^{-13} \mathrm{~F}\right)$

\begin{tabular}{cccc}
\hline Position & $\mathbf{C m a x}(\mathbf{F})$ & Tunability & Tuning ratio \\
\hline$\chi_{d}=0.3$ & $3.96 \times 10^{-13} F$ & $42.96 \%$ & $142.96 \%$ \\
$\chi_{d}=0.6$ & $6.94 \times 10^{-13} F$ & $149.73 \%$ & $250.54 \%$ \\
$\chi_{d}=0.9$ & $27.78 \times 10^{-13} F$ & $899.64 \%$ & $999.64 \%$
\end{tabular}

of this device. In the following, the tunability of the system is examined in relation to implementation of a controller. The tunability and tuning ratio are defined as follows 41]:

Tunability $=\frac{\left(C_{\max }-C_{\min }\right)}{C_{\min }} \times 100$

Tuning ratio $=\frac{C_{\max }}{C_{\min }} \times 100$

It is obtained that the minimum capacitance for the proposed CMUT is equal to $2.779 \times 10^{-13}(F)$. However, due to control technique which is proposed in this work, minimal distance between two electrodes can reach to value of $0.05 \mu \mathrm{m}$ and consequently a maximum capacitance of $5.55 \times 10^{-12}(F)$ which is presented in Fig. 5 a.

For various values of $\chi_{d}$, Table 2 summarizes the maximum capacitance, tunability, and tuning ratio and as can be seen that using the proposed adaptive sliding mode technique, an acceptable range of tunability and tuning ratio are obtained. 
Furthermore, Fig. 8a and b illustrate the time history of capacitance and output pressure of the CMUT for different $\chi_{d}$. The time history of different capacitance considering various $\chi_{d}$ are plotted in Fig. 8p in which it can be seen that the obtained results are compatible with calculated values in Table. 2, Also, it is known that the output pressure determines the penetration depth in ultrasound applications and it is considered as a function of time [47. In this figure it is shown that a large displacement is desired for increasing output pressure. Thus, increasing the displacement can have a positive influence on the key features of a CMUT including output pressure and tunability.

\section{Conclusion}

A fully clamped micro-scale Kirchhoff plate as a movable plate and main mechanical part of a CMUT is discussed. To seize a maximum displacement, an adaptive sliding mode controller is proposed and investigated to control the CMUT. Using a capacitor control, its aim is to move the deformable electrode to a closer distance from fixed electrode and therefore increase the capacitor's overall maximum capacitance as well as its tuning capability. Moreover, in order to eliminate the adverse effects of uncertainties, unmodeled dynamics and external disturbances potential of the designed adaptive robust sliding mode control strategy was evaluated. Ac-
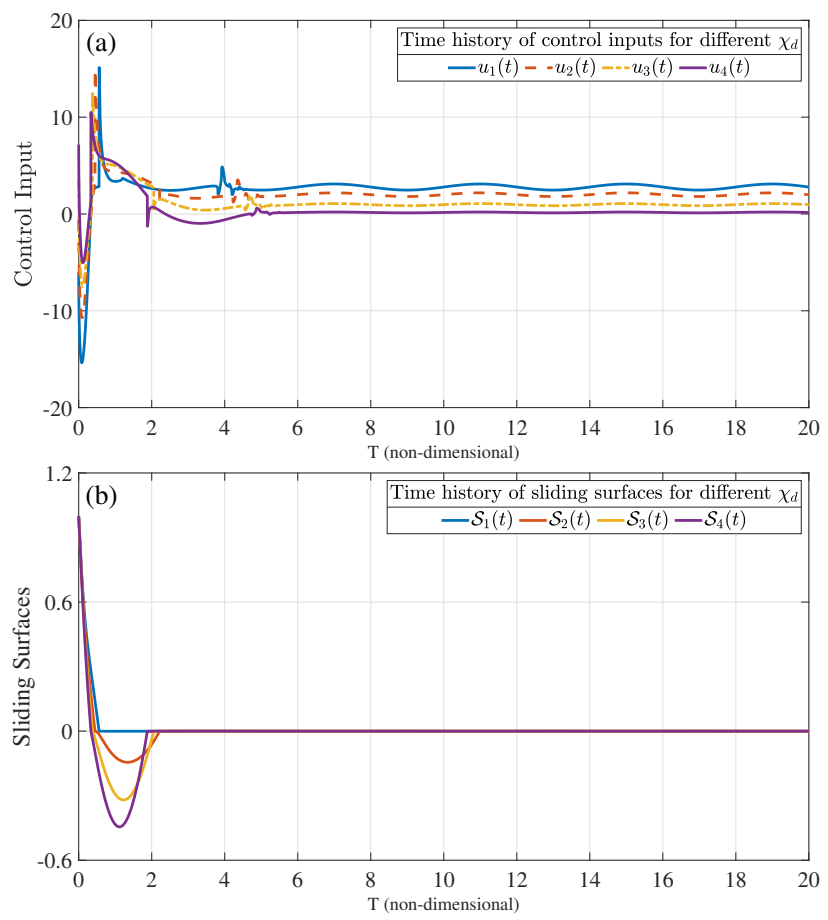

Fig. 7 The variation of a) control input and b) sliding surface versus time for various values of $\chi_{d}$
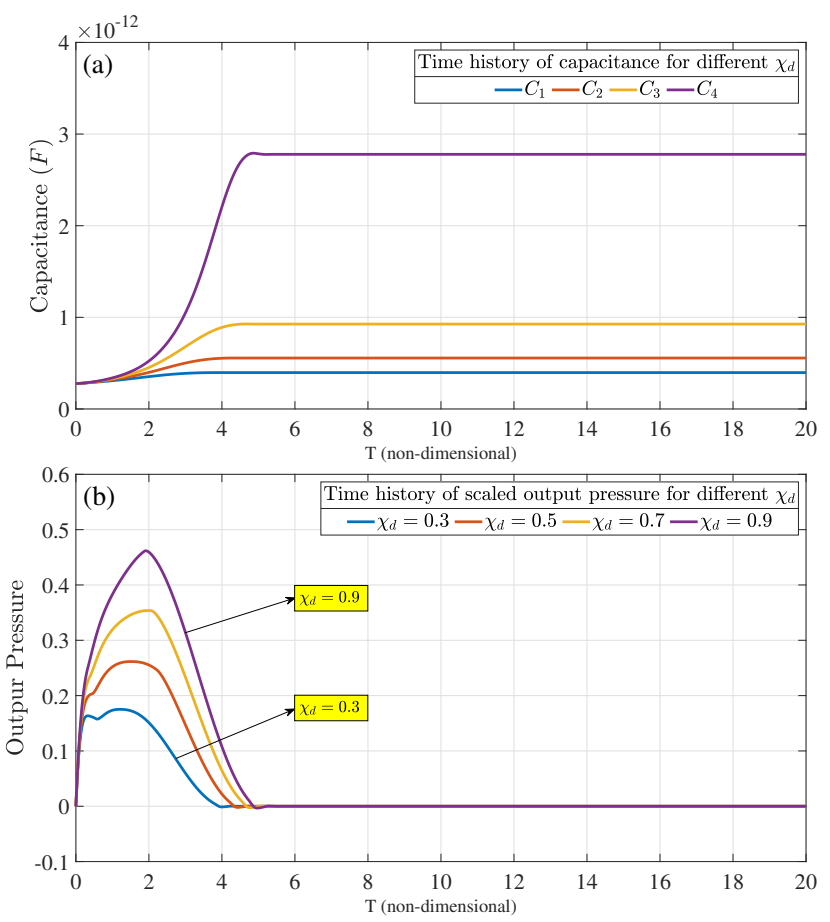

Fig. 8 The variation of a) capacitance and b) output pressure versus time for various values of $\chi_{d}$.

cording to the results of evaluation, the controller provides a high robustness and stable performance, and compensates anomalies. The functionality of controller in terms of improving output pressure, stabilizing the CMUT and its robustness was investigated. In the last part, It is shown that a CMUT a with wide displacement ranges can have more desirable characteristics, such as a higher output pressure and tunability.

\section{Compliance with ethical standards}

Funding Not applicable.

Conflict of interest The authors declare that they have no conflict of interest.

Availability of data and material Data will be made available on reasonable request.

Code availability Data will be made available on reasonable request.

Ethics approval Not applicable.

Consent to participate Not applicable.

Consent for publication We, the undersigned, give our consent for the publication of identifiable details, which can include photograph(s) and/or details within the text to be published in the above journal and article. 


\section{References}

1. Rembe C, Muller RS (2002) Measurement system for full three-dimensional motion characterization of mems. Journal of Microelectromechanical Systems 11(5):479-488

2. Joshitha C, Sreeja B, Princy SS, Radha S (2017) Fabrication and investigation of low actuation voltage curved beam bistable mems switch. Microsystem Technologies 23(10):4553-4566

3. Ghasemi S, Afrang S, Rezazadeh G, Darbasi S, Sotoudeh B (2020) On the mechanical behavior of a wide tunable capacitive mems resonator for low frequency energy harvesting applications. Microsystem Technologies pp 1-10

4. Maillard D, De Pastina A, Abazari AM, Villanueva LG (2021) Avoiding transduction-induced heating in suspended microchannel resonators using piezoelectricity. Microsystems \& Nanoengineering $7(1): 1-7$

5. Li Q, Fan S, Tang Z, Xing W (2012) Non-linear dynamics of an electrothermally excited resonant pressure sensor. Sensors and Actuators A: Physical 188:19-28

6. Fang S, Wang S, Miao G, Zhou S, Yang Z, Mei X, Liao WH (2020) Comprehensive theoretical and experimental investigation of the rotational impact energy harvester with the centrifugal softening effect. Nonlinear Dynamics 101(1):123-152

7. Fang S, Wang S, Zhou S, Yang Z, Liao WH (2020) Analytical and experimental investigation of the centrifugal softening and stiffening effects in rotational energy harvesting. Journal of Sound and Vibration 488:115643

8. Safavi SM, Rezazadeh G, Fathalilou M, Abazari AM (2016) Couple stress effect on micro/nanocantilever-based capacitive gas sensor. International Journal of Engineering 29(6):852-861

9. Abazari AM, Fotouhi M, Tavakkoli H, Rezazadeh G (2020) An experimental study for characterization of size-dependence in microstructures via electrostatic pull-in instability technique. Applied Physics Letters 116(24):244102

10. Jie L, Wenjun W, Shan X, Fang C (2019) Effect of structural parameters on functional performance of cmut: a simulation study and analysis. In: 2019 14th IEEE International Conference on Electronic Measurement \& Instruments (ICEMI), IEEE, pp 936-941

11. Fang S, Wang S, Zhou S, Yang Z, Liao WH (2020) Exploiting the advantages of the centrifugal softening effect in rotational impact energy harvesting. Applied Physics Letters 116(6):063903
12. Mirzaei MJ, Aslmostafa E, Asadollahi M, Badamchizadeh MA, Ghaemi S (2020) Fuzzy terminal sliding mode control for finite-time synchronization of electrostatic and electromechanical transducers. In: 2020 6th Iranian Conference on Signal Processing and Intelligent Systems (ICSPIS), IEEE, pp 1-7

13. Sharma R, Agarwal R, Dubey AK, Arora A (2019) Design and analysis of capacitive micromachined ultrasonic transducer. Recent Patents on Engineering 13(2):108-116

14. Darbasi S, Shourcheh SD, Rezazadeh G, Afrang S (2018) Mechanical behavior of a capacitive tunable ultrasound transducer for bio diagnostic application. In: Electrical Engineering (ICEE), Iranian Conference on, IEEE, pp 336-341

15. Son C, Ziaie B (2008) Pull-in instability of parallelplate electrostatic microactuators under a combined variable charge and voltage configuration. Applied Physics Letters 92(1):013509

16. Yaralioglu GG, Ergun AS, Bayram B, Haeggstrom E, Khuri-Yakub BT (2003) Calculation and measurement of electromechanical coupling coefficient of capacitive micromachined ultrasonic transducers. IEEE transactions on ultrasonics, ferroelectrics, and frequency control 50(4):449-456

17. Vogl GW (2006) Nonlinear dynamics of circular plates under electrical loadings for capacitive micromachined ultrasonic transducers (cmuts). PhD thesis, Virginia Tech

18. Pekař M, Dittmer WU, Mihajlović N, van Soest G, de Jong N (2017) Frequency tuning of collapsemode capacitive micromachined ultrasonic transducer. Ultrasonics 74:144-152

19. Owusu KO, Lewis FL (2007) Solving the" pull-in" instability problem of electrostatic microactuators using nonlinear control techniques. In: 2007 2nd IEEE International Conference on Nano/Micro Engineered and Molecular Systems, IEEE, pp 11901195

20. Michael SCL, Gary K (2002) Closed-loop control of a parallel-plate microactuator beyond the pull-in limit. In: Technical Digest of the Solid-State Sensor, Actuator and Microsystems Workshop, pp 255-258

21. Trivedi R, Pawaskar D, Shimpi R (2021) Enhancement of travel range of electrostatically driven fixed-fixed microbeam in static and dynamic mode using mutated particle swarm optimization. Structural and Multidisciplinary Optimization pp 1-17

22. Qin Y, Sun W, Yeow JT (2019) A robust control approach for mems capacitive micromachined ultrasonic transducer. Transactions of the Institute of Measurement and Control 41(1):107-116 
23. Zhao Z, Liu Y, Guo F, Fu Y (2017) Vibration control and boundary tension constraint of an axially moving string system. Nonlinear dynamics 89(4):2431-2440

24. Lu MC, Fedder GK (2004) Position control of parallel-plate microactuators for probe-based data storage. Journal of microelectromechanical Systems 13(5):759-769

25. Chan EK, Dutton RW (2000) Electrostatic micromechanical actuator with extended range of travel. Journal of microelectromechanical Systems 9(3):321-328

26. Zhu G, Lévine J, Praly L, Peter YA (2006) Flatness-based control of electrostatically actuated mems with application to adaptive optics: A simulation study. Journal of Microelectromechanical Systems 15(5):1165-1174

27. Indeitsev D, Belyaev YV, Lukin A, Popov I (2021) Nonlinear dynamics of mems resonator in pll-agc self-oscillation loop. Nonlinear Dynamics pp 1-18

28. Mousavi M, Alzgool M, Towfighian S (2021) Electrostatic levitation: an elegant method to control mems switching operation. Nonlinear Dynamics pp $1-17$

29. Ruzziconi L, Jaber N, Kosuru L, Bellaredj ML, Younis MI (2021) Internal resonance in the higherorder modes of a mems beam: experiments and global analysis. Nonlinear Dynamics 103(3):21972226

30. Pisano A, Usai E (2011) Sliding mode control: A survey with applications in math. Mathematics and Computers in Simulation 81(5):954-979

31. Ye M, Wang H, Yazdani A, He S, Ping Z, Xu W (2021) Discrete-time integral terminal sliding mode-based speed tracking control for a robotic fish. Nonlinear Dynamics pp 1-12

32. Chen L, Liu J, Wang H, Hu Y, Zheng X, Ye M, Zhang J (2021) Robust control of reaction wheel bicycle robot via adaptive integral terminal sliding mode. Nonlinear Dynamics pp 1-12

33. Liu X, Yu H (2021) Continuous adaptive integraltype sliding mode control based on disturbance observer for pmsm drives. Nonlinear Dynamics 104(2):1429-1441

34. Aslmostafa E, Mirzaei MJ, Asadollahi M, Badamchizadeh MA (2021) Synchronization problem for a class of multi-input multi-output systems with terminal sliding mode control based on finite-time disturbance observer: Application to chameleon chaotic system. Chaos, Solitons \& Fractals 150:111191

35. Utkin V, Shi J (1996) Integral sliding mode in systems operating under uncertainty conditions. In:
Proceedings of 35th IEEE conference on decision and control, IEEE, vol 4, pp 4591-4596

36. Castaños F, Fridman L (2006) Analysis and design of integral sliding manifolds for systems with unmatched perturbations. IEEE Transactions on Automatic Control 51(5):853-858

37. Bandyopadhyay B, Deepak F, Park Y (2008) A robust algorithm against actuator saturation using integral sliding mode and composite nonlinear feedback. IFAC Proceedings Volumes 41(2):1417414179

38. Mirzaei MJ, Aslmostafa E, Asadollahi M, Badamchizadeh MA (2021) Robust adaptive finitetime stabilization control for a class of nonlinear switched systems based on finite-time disturbance observer. Journal of the Franklin Institute 358(7):3332-3352

39. Shi S, Feng H, Liu W, Zhuang G (2019) Finite-time consensus of high-order heterogeneous multi-agent systems with mismatched disturbances and nonlinear dynamics. Nonlinear Dynamics 96(2):13171333

40. Zhang R, Xu B, Zhao W (2020) Finite-time prescribed performance control of mems gyroscopes. Nonlinear Dynamics pp 1-12

41. Mobki H, Sabegh AM, Azizi A, Ouakad HM (2020) On the implementation of adaptive sliding mode robust controller in the stabilization of electrically actuated micro-tunable capacitor. Microsystem Technologies pp 1-14

42. Koymen H, Atalar A, Aydogdu E, Kocabas C, Oguz HK, Olcum S, Ozgurluk A, Unlugedik A (2012) An improved lumped element nonlinear circuit model for a circular cmut cell. IEEE transactions on ultrasonics, ferroelectrics, and frequency control 59(8):1791-1799

43. Brock WA, Hsieh DA, LeBaron BD, Brock WE, et al. (1991) Nonlinear dynamics, chaos, and instability: statistical theory and economic evidence. MIT press

44. Najar F, Nayfeh A, Abdel-Rahman E, Choura S, El-Borgi S (2010) Nonlinear analysis of mems electrostatic microactuators: primary and secondary resonances of the first mode. Journal of Vibration and Control 16(9):1321-1349

45. Bhat SP, Bernstein DS (2005) Geometric homogeneity with applications to finite-time stability. Mathematics of Control, Signals and Systems 17(2):101-127

46. Saadatmand M, Kook J (2020) Differences between plate theory and lumped element model in electrostatic analysis of one-sided and two-sided cmuts with circular microplates. Journal of the Brazil- 
ian Society of Mechanical Sciences and Engineering 42(9):1-11

47. Lin DS (2011) Interface engineering of capacitive micromachined ultrasonic transducers for medical applications. Stanford University 